\title{
Transient Hypothyroidism and Autoimmune Thyroiditis in Children With Chronic Hepatitis C Treated With Pegylated-interferon- $\alpha-2 b$ and Ribavirin
}

\author{
Daniele Serranti, MD, * Giuseppe Indolfi, MD, * Gabriella Nebbia, MD, † Mara Cananzi, MD, $\neq$ \\ Lorenzo D’Antiga, MD, \$ Silvia Ricci, MD, $\mid$ Stefano Stagi, MD, $\mid$ Chiara Azzari, MD, PhD, $\mid$ \\ and Massimo Resti, MD, * for the Italian Study Group for Treatment of Chronic Hepatitis $C$ in Children
}

\begin{abstract}
Background: Autoimmune thyroid disease and thyroid dysfunction are common in adults receiving interferon (IFN)-based treatment for chronic hepatitis $\mathrm{C}(\mathrm{CHC})$. Few data are available in children with $\mathrm{CHC}$. This study is aimed to evaluate the appearance and timing of thyroid dysfunction and antithyroid autoimmunity in children with $\mathrm{CHC}$ treated with pegylated IFN$\alpha-2 b$ and ribavirin (RBV).

Methods: Sixty-one otherwise healthy children with $\mathrm{CHC}, 3-17$ years of age, infected perinatally and treatment naïve, receiving therapy with pegylated IFN- $\alpha-2 b$ and RBV and 183 age- and sex-matched controls were included in a multicenter, prospective, case-control study. Thyroid-stimulating hormone, free thyroxine, antithyroglobulin antibodies and antithyroid peroxidase antibodies were assessed before, during and 24 weeks after the end of treatment. Results: From baseline to the end of treatment, subclinical hypothyroidism and autoimmune thyroiditis were diagnosed in 17 of $61(27.94 \%)$ and in 4 of $61(6.6 \%)$ of the children treated, respectively, and in 5 of $183(2.7 \%)$ and in none of the controls $(P<0.0001$, relative risk: $10.2,95 \%$ confidence interval: $3.9-26.5 ; P=0.03$, relative risk: $26.8,95 \%$ confidence interval: 1.5-489.1, respectively). Twenty-four weeks after the end of treatment, subclinical hypothyroidism persisted in only 4 of 61 (6.6\%). Autoimmune thyroiditis persisted in 3 of 4 ( $75 \%$ ) of the cases.

Conclusions: Subclinical hypothyroidism is common in children with $\mathrm{CHC}$ receiving treatment with pegylated IFN- $\alpha-2 b$ and $R B V$, but in most cases is transient. Autoimmune thyroiditis, which is less common, generally persists after treatment completion. Thyroid function should be carefully monitored in patients presenting with antithyroid autoantibodies and thyroid dysfunction during and after pegylated IFN- $\alpha$-based treatment.
\end{abstract}

Key Words: hypothyroidism, interferon, hepatitis C, autoimmunity, children

(Pediatr Infect Dis J 2018;37:287-291)

nterferon (IFN)- $\alpha$ is a cytokine belonging to type I IFNs produced in vivo by the innate immune system after infections and recognition of pathogens. ${ }^{1}$ IFN- $\alpha$ and its pegylated form (peg-IFN $\alpha$ ) has

Accepted for publication July 2, 2017.

From the *Paediatric and Liver Unit, Meyer Children's University Hospital of Florence, Florence, †Fondazione IRCCS Ca' Granda Ospedale Maggiore Policlinico, Milano, +University Hospital of Padova, Padova, §Hospital Papa Giovanni XXIII, Bergamo, and University of Florence, Florence, Italy.

The authors have no funding or conflicts of interest to disclose.

D.S., G.I., G.N., M.C., L.D., S.S., C.A. and M.R. created the project. D.S., G.I., S.R. and S.S. collected the data, wrote the first draft of the article, critically revised it and gave the final approval to submission. G.N., L.D'., C.A. and M.R., as experts, critically revised the article and gave the final approval to submission.

Address for correspondence: Giuseppe Indolfi, MD, Paediatric and Liver Unit, Meyer Children's University Hospital of Florence, Viale Gaetano Pieraccini 24, Firenze 50139, Italy. E-mail: g.indolfi@meyer.it.

Supplemental digital content is available for this article. Direct URL citations appear in the printed text and are provided in the HTML and PDF versions of this article on the journal's website (www.pidj.com).

Copyright (C) 2017 Wolters Kluwer Health, Inc. All rights reserved.

ISSN: 0891-3668/18/3704-0287

DOI: $10.1097 /$ INF.0000000000001791 been used for treatment of patients with cancer and chronic viral infections. ${ }^{1}$ IFN- $\alpha$-based treatments, despite their well-known beneficial therapeutic effects, have been associated with many adverse events and, among these, with the possible development of autoimmune phenomena. ${ }^{1}$

The combination of peg-IFN $\alpha$ and ribavirin (RBV) has been for a long time the standard of care for treatment of adults with chronic hepatitis $\mathrm{C}(\mathrm{CHC})$ and is still the only available therapeutic option for children. ${ }^{2}$ Peg-IFN $\alpha$ and RBV lead to the resolution of the infection in $89 \%$ of children with hepatitis $\mathrm{C}$ virus (HCV) genotype 2 or 3 infection and $52 \%$ of those with genotypes 1 and $4 .^{3}$ The appearance of autoantibodies and new onset of autoimmune diseases have been described in adults ${ }^{4}$ and children ${ }^{5}$ during and after IFN- $\alpha$-based treatment. Autoimmune thyroid disease and thyroid dysfunction were described in $20 \%-40 \%$ and $11 \%-15 \%$, respectively, of the adults with $\mathrm{CHC}$ receiving IFN. ${ }^{6}$ Two more recently published studies, based on large cohorts of patients, demonstrate that adults with $\mathrm{CHC}$ treated with peg-IFN $\alpha$ and RBV present a high incidence of thyroid disease during treatment (35\% $\%^{7}$ and $27.7 \%,{ }^{8}$ respectively) with most of them recovering after completion of therapy. The exact mechanism explaining the relation between IFN- $\alpha$ and thyroid disease is not clear. An immunemediated mechanism has been hypothesized. IFN- $\alpha$ modulates the production of other cytokines, like IFN- $\gamma$ and IL- 6 , and creates an imbalance of the immune system which leads to the production of thyroid autoantibodies and to a direct disruptive effect on thyroid function, at the same time. ${ }^{6,9}$ Untreated children with $\mathrm{CHC}$ are at risk of both autoimmune and nonautoimmune thyroid disease with subclinical hypothyroidism and autoimmune thyroiditis described, respectively, in $11.1 \%$ and $2.8 \%$ of treatment-naïve children with chronic infection. ${ }^{10}$ Few data are available on the effects of the association of peg-IFN $\alpha$ and RBV on thyroid function and antithyroid autoimmunity in children with $\mathrm{CHC} .{ }^{11-17}$ Previous studies provided different figures and were variably biased by the low number of children enrolled, ${ }^{12,15,16}$ the different sources of infection ${ }^{11-14,16,17}$ and treatment histories ${ }^{11,12,15,16}$ and the possible presence of comorbidities. ${ }^{15,16}$ Furthermore, thyroid function and autoantibodies were not systematically assessed in every study.

The main aim of the present multicenter, prospective study was to evaluate systematically in a large and homogeneous cohort of treatment-naïve, vertically infected children with $\mathrm{CHC}$ treated with peg-IFN $\alpha-2 b$ and RBV the appearance and timing of thyroid dysfunction and antithyroid autoimmunity.

\section{MATERIALS AND METHODS}

\section{Patients}

A multicenter prospective study on the evaluation of safety and efficacy of treatment with peg-IFN $\alpha-2 b$ and RBV was performed enrolling Italian children with $\mathrm{CHC}^{18}$ from 11 different Italian centers [Florence, Milan, Padua, Pavia (2 centers), Trieste, Ancona, Turin, Bergamo, Parma, Trento] between January 2012 and 
December 2015. Children were included if between 3 and 17 years of age, infected perinatally and treatment naïve. Perinatal infection was defined by the detection of HCV RNA in peripheral blood by reverse transcriptase polymerase chain reaction or when anti-HCV antibodies persisted beyond 18 months of age ${ }^{19} \mathrm{CHC}$ was defined by the presence of positive anti-HCV antibodies and HCV RNA as detected by reverse transcriptase polymerase chain reaction in at least 2 serum samples taken 6 months apart. ${ }^{18}$ The main exclusion criteria included body weight $>90 \mathrm{~kg}$, low neutrophils $(<1.500 / \mu \mathrm{L})$ and platelets count $(<100.000 / \mu \mathrm{L})$, low hemoglobin level $(<11 \mathrm{~g} /$ $\mathrm{dL}$ for female, $<12 \mathrm{~g} / \mathrm{dL}$ for male), high creatinine ( $>1.5$ times the age-appropriate normal value), presence of advanced liver disease, coexisting human immunodeficiency virus or hepatitis B virus infection, diagnosis of autoimmune hepatitis or presence of autoantibodies (liver-kidney microsomal type-1 autoantibody, smooth muscle autoantibody $>1: 100$, antinuclear antibodies $>1: 160$ with homogeneous pattern) and/or of other systemic diseases. ${ }^{20}$

\section{Controls}

HCV-infected children were compared with a control group composed of 183 age- and sex-matched Italian children ( 94 females: $51.4 \%$, 89 males: $48.6 \%$, median age: 12.6 , range: $3.1-16.9$ years), admitted to the Meyer Children's University Hospital for minor surgical interventions (eg, adenotonsillectomy, phimosis, dermoid cyst, herniotomy). Part of this group was previously reported..$^{21}$ In all controls, HCV infection was excluded at the time of the study.

\section{Treatment}

Treatment consisted of peg-IFN $\alpha-2 b(1.5 \mu \mathrm{g} / \mathrm{kg} / \mathrm{wk})$ onceweekly plus RBV $(15 \mathrm{mg} / \mathrm{kg} / \mathrm{d}$ up to a maximum of $1200 \mathrm{mg} / \mathrm{d})$. Children with genotype 1 or 4 were assigned to 48 weeks of treatment but, if HCV RNA was detectable at week 24, they discontinued treatment and entered the follow-up phase. All children with genotype 2 or 3 were assigned to 24 weeks of treatment. All patients were followed up for 24 weeks after the end of treatment.

\section{Laboratory Investigations}

Thyroid-stimulating hormone (TSH), serum free thyroxine (FT4), antithyroglobulin antibodies (anti-TG) and antithyroid peroxidase antibodies (anti-TPO) were assessed on blood samples by commercially available immunometric assays. Results exceeding the upper normal value reported for each test were considered positive. TSH, FT4, anti-TG and anti-TPO were assessed at baseline and during treatment at 4, 8, 12, 18 and 24 weeks for all genotypes and also at 36 and 48 weeks only for genotypes 1 and 4, respectively. TSH, FT4, anti-TG and anti-TPO dosages were performed in all patients at 4, 12 and 24 weeks after completion of treatment.

\section{Definitions}

Subclinical hypothyroidism was defined as a TSH level higher than $4 \mathrm{mUI} / \mathrm{L}$, together with normal serum thyroid hormone levels. ${ }^{22-24}$ Overt hypothyroidism was defined as increased TSH and decreased serum thyroid hormone level. ${ }^{22,23,25}$ Autoimmune thyroiditis was defined by raised anti-TPO and/or anti-TG values, and raised TSH levels and/or typical hypoechogenicity of the thyroid ultrasound (US). ${ }^{22,23,25}$ The thyroid was considered hypoechogenic when its signal was equal to or below the echogenicity of the surrounding neck muscles. ${ }^{22,23,25}$ Sustained virologic response (SVR) was defined as undetectable plasma HCV RNA 24 weeks after the end of treatment. ${ }^{18}$

\section{Statistical Analysis}

Data were processed with the IBM SPSS Statistics for Macintosh release 21 statistical package. Results were expressed as median and interquartile range. The Mann-Whitney $U$ test was used to compare continuous variables between 2 groups. Chi-square test and Fisher exact test were performed to test for differences in proportions of categorical variables between 2 groups. Relative risk (RR) with $95 \%$ confidence intervals (CIs) was calculated. The level of $P<0.05$ was considered significant.

\section{RESULTS}

Sixty-one treatment-naïve children with $\mathrm{CHC}$ were enrolled. Genotype distribution was as following: 21 (32.8\%) genotype 1, 16 (24.6\%) genotype 2, 22 (34.4\%) genotype 3 and 5 (8.2\%) genotype 4. According to genotypes, SVR was $47.6 \%(10 / 21)$ for genotype 1 , $100 \%(16 / 16)$ for genotype $2,95.5 \%(21 / 22)$ for genotype $3,80 \%$ (4/5) for genotype 4 , respectively.

\section{Hypothyroidism and Autoimmune Thyroiditis}

From baseline to the end of therapy, 17 children (27.9\%) were diagnosed with subclinical hypothyroidism and none with overt hypothyroidism.

Autoimmune thyroiditis was diagnosed in 4 children (6.6\%). In this latter group, all the children presented with increased TSH, positive autoantibodies ( 4 anti-TG and 3 anti-TPO) and 3 had abnormal US. The development of any thyroid disease (subclinical or overt hypothyroidism and autoimmune thyroiditis) did not correlate with age, sex, weight, height and baseline viral load of the patients (Table 1).

\section{Control Group}

Five of 183 (2.7\%) subjects had subclinical hypothyroidism with negative antithyroid autoantibodies; no child was diagnosed with overt hypothyroidism or autoimmune thyroiditis. Two (1.1\%) subjects showed isolated high titers of antithyroid autoantibodies with normal thyroid function and thyroid US.

\section{HCV-infected Children Versus Controls}

Subclinical hypothyroidism was more frequent in HCVinfected children receiving therapy than controls $(P<0.0001, \mathrm{RR}$ :

TABLE 1. Characteristics of Patients Presenting Thyroid Disease Compared With Those With Normal Thyroid Function

\begin{tabular}{lccrr}
\hline \hline & All & Any Thyroid Disease & Normal Thyroid Function & $P$ \\
\hline $\mathrm{N}$ & 61 & 21 & 40 & $12.7(5.9)$ \\
Age (yr), median (IQR) & $12.8(5.6)$ & $13.2(5.4)$ & $23(53.5)$ & 0.84 \\
Male, n (\%) & $30(49.2)$ & $7(38.9)$ & $46.3(25.4)$ & 0.86 \\
Weight (kg), median (IQR) & $46(26.1)$ & $153(24.3)$ & $154(25.8)$ & 0.98 \\
Height (cm), median (IQR) & $154.5(25.8)$ & $233,500(1,492,374)$ & $272,000(1,279,139)$ & 0.80 \\
Baseline viral load & $284,000(1,054,630)$ & & \\
$\quad$ (IU/mL), median (IQR) & & & & \\
\hline
\end{tabular}

$\mathrm{IQR}$ indicates interquartile range. 
10.2, 95\% CI: 3.9-26.5). No patient presented overt hypothyroidism in the 2 groups. Autoimmune thyroiditis prevalence was higher in HCV-infected children receiving therapy compared with controls $(P=0.0268$, RR: $26.8,95 \%$ CI: $1.5-489.1)$.

\section{Timing of Hypothyroidism and Autoimmune Thyroiditis}

At baseline, 2 (3.3\%) children had subclinical hypothyroidism while no patient was positive for antithyroid autoantibodies. During treatment, the highest number of patients with thyroid disease was found at week 24 when $10(16.4 \%)$ children presented subclinical hypothyroidism. Twenty-four weeks after the end of treatment, subclinical hypothyroidism persisted in only 4 children.

The TSH trends for each patient with subclinical hypothyroidism, and the TSH median values with ranges have been reported in Supplemental Digital Content 1, http://links.lww.com/ INF/C845.

Six $(9.8 \%)$ children became positive for antithyroid autoantibodies during treatment. Two $(3.3 \%)$ of them were positive only once at weeks 8 and 18 ( 1 with anti-TG and 1 with anti-TPO), respectively, and did not show any abnormality in TSH level or thyroid US. The remaining 4 (6.6\%) were diagnosed with autoimmune thyroiditis at week 18, which persisted at the last follow-up visit in 3 of them. Of these 4 patients, 1 presented transient autoimmune thyroiditis, as was positive for anti-TG from treatment week 4-8, presented raised TSH from treatment week 8-36 and had a normal thyroid US examination. The remaining 3 became positive both for anti-TG and anti-TPO at weeks 12, 18 and 24, respectively, and presented thyroid hypoechogenicity at US. They were started and are still on treatment with thyroxine replacement therapy. At the end of follow-up, 2 of them (3.3\%) were still positive for antithyroid autoantibodies and 1 had negative antibodies.

\section{DISCUSSION}

The present study shows that thyroid dysfunction is common in children with $\mathrm{CHC}$ treated with peg-IFN $\alpha$ and RBV. In the described cohort, subclinical hypothyroidism and autoimmune thyroiditis were diagnosed in $27.9 \%$ and $6.6 \%$ of the children treated, respectively. Subclinical hypothyroidism was transient in $13(76.5 \%)$ of 17 cases, respectively. Autoimmune thyroiditis persisted 24 weeks after the end of treatment in $3(75 \%)$ of 4 children.

The present results provide additional knowledge to previous studies evaluating the effects of peg-IFN $\alpha$ and RBV therapy on thyroid function in children (Table 2). The cohort of children enrolled in this study was highly homogeneous being all the children infected vertically, treatment naïve, with no comorbidity and specifically with no preexisting autoimmune disease. Moreover, thyroid function and thyroid autoantibodies were systematically evaluated to detect the exact timing of appearance of TSH abnormalities and anti-TG and/or anti-TPO positivities. Such a strict monitoring of thyroid function and autoantibodies in children with $\mathrm{CHC}$ treated with peg-IFN $\alpha$ and RBV was never reported. Previous studies reported prevalence rates of subclinical and overt hypothyroidism ranging between $0 \%-23 \%$ and $0 \%-5 \%$, respectively. ${ }^{11-17}$ In the present study, subclinical hypothyroidism was diagnosed in $27.9 \%$ of patients. This result is higher than previously reported in other studies performed in larger as well as smaller cohorts of children. Only the study of Wirth et $\mathrm{al}^{13}$ in 2010 , based on a large cohort of 107 children, found a similar, slightly lower prevalence (Table 2). It is possible to speculate that in all the previous studies, the results could have been affected by a less intense monitoring schedule or by the lower number of patients (Table 2).

Whether the correlation between IFN-based treatment and thyroid dysfunction is a direct consequence of therapy and/or it depends on HCV infection is matter of debate. In a previous study, the prevalence of subclinical hypothyroidism was $11.1 \%$ in a cohort of $\mathrm{HCV}$ vertically infected, treatment-naïve children. ${ }^{10}$ In the present study, the prevalence of subclinical hypothyroidism was more than double (27.9\%). These independent observations suggest that both IFN-based treatment and HCV infection play an important role in the development of thyroid dysfunction in children with CHC.

The scenario depicted by the results of the present study allows to define more clearly what could be the approach to patients presenting thyroid dysfunction and/or thyroid autoantibodies during treatment with peg-IFN $\alpha$ and RBV. Children should not be routinely tested for antithyroid autoantibodies, but only if a raised TSH is detected. Those children presenting subclinical hypothyroidism should be reassured that in most instances, this is only a transient event which usually recovers. Thyroid function should be monitored, but progression of the disease is unlikely and replacement therapy with thyroxine generally is not needed. On the other side, children positive for antithyroid autoantibodies and with raised TSH need an accurate endocrine follow-up, including thyroid US scan, because autoimmune thyroiditis usually persists after the completion of treatment.

Peg-IFN $\alpha$ is no longer the standard of care in adults with $\mathrm{CHC}$ with the arrival of the new highly effective and safe directacting antiviral agents. ${ }^{26}$ Recently, a trial on the use of ledipasvirsofosbuvir in adolescents 12-17 years old with CHC showed promising results with $98 \%$ of patients reaching SVR at 12 weeks after treatment. ${ }^{27}$ The fixed dose combination of ledipasvir/sofosbuvir and the association of sofosbuvir and RBV have been approved recently by the Food and Drug Administration for the treatment of children 12-17 years old with $\mathrm{CHC}$ infected by genotypes $1,4,5$ or 6 and genotypes 2 and 3, respectively. ${ }^{28}$ Hopefully, in the future, these drugs as well as other direct-acting antivirals combinations will be approved also children younger than 12 years. ${ }^{29}$ By the

TABLE 2. Thyroid Function and Thyroid Autoantibodies in Children With CHC Treated With Pegylated-interferon- $\alpha$, Summary of the Results of Previous Studies

\begin{tabular}{|c|c|c|c|c|c|}
\hline Authors & $\mathrm{N}$ & Increased TSH (\%) & Hyperthyroidism (\%) & Hypothyroidism (\%) & Autoantibodies (\%) \\
\hline Wirth et $\mathrm{al}^{11}$ & 62 & $6(10)$ & 0 & 0 & $7(11)$ \\
\hline 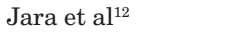 & 30 & $6(20)$ & $2(7)$ & 0 & $4(13)$ \\
\hline Wirth et $\mathrm{al}^{13}$ & 107 & $25(23)$ & 0 & $3(3)$ & Not reported \\
\hline Sokal et $\mathrm{al}^{14}$ & 65 & 7 (11) & $1(2)$ & 0 & Not reported \\
\hline Baker et al $^{15}$ & 10 & 0 & 0 & 0 & 0 \\
\hline Tajiri et al $^{16}$ & 33 & Not reported & 0 & $2(5)$ & $6(16)$ \\
\hline $\begin{array}{l}\text { Molleston et al and } \\
\text { Schwarz et al }\end{array}$ & 114 & Not reported & 0 & $2(2)$ & $\begin{array}{c}\text { Anti-TG } 4(6.5) \\
\text { Anti-TPO } 2(3.2)\end{array}$ \\
\hline Indolfi et $\mathrm{al}^{10}, *$ & 36 & $4(11.1)$ & 0 & 0 & $2(5.6)$ \\
\hline
\end{tabular}

*Included only untreated children. 
time, peg-IFN $\alpha$ and RBV will still be the only treatment available for children with $\mathrm{CHC}$ between 3 and 11 years of age. Furthermore, IFN- $\alpha$ is still a mainstay of therapy for chronic hepatitis B in children. ${ }^{30-32}$ Caregivers should be aware of the possible thyroid involvement in children undergoing treatment with peg-IFN $\alpha$ and RBV. A better knowledge about the long-term consequences of thyroid dysfunction and appearance of antithyroid autoantibodies during treatment of $\mathrm{CHC}$ in children is mandatory to assure their correct management.

The present study is not free of limitations. The multicenter design did not allow testing blood samples for thyroid function and antithyroid autoantibodies in the same laboratory with only 1 assay. Samples were tested with commercially available kits, which have been verified to be in line with the standard. The definition of subclinical hypothyroidism used in this study, although widely accepted, being based on a single determination of TSH could probably overestimate its real prevalence. ${ }^{24}$ The follow-up of the children treated was reported up to 24 weeks after treatment completion and is still ongoing. The possible late onset thyroid dysfunction or late appearance of antithyroid autoantibodies cannot be excluded, and a longer follow-up after treatment is recommended in clinical practice.

This study demonstrates that thyroid dysfunction is common in children treated with peg-IFN $\alpha$ and RBV for CHC. Subclinical hypothyroidism is the most common manifestation, but in most cases is transient. By comparison, autoimmune thyroiditis is less frequent, but generally is a permanent disease. Clinicians who decide to administer peg-IFN $\alpha$-based treatment to children with CHC should carefully evaluate antithyroid autoantibody appearance and thyroid function during treatment and follow-up to promptly detect any thyroid dysfunction and establish the proper management.

\section{ACKNOWLEDGMENTS}

We are grateful to all the members of the Italian Study Group for Treatment of Chronic Hepatitis $C$ in Children who gave a substantial contribution to realize this study: Roberto Guidi, MD, Azienda Ospedaliero-Universitaria Ospedali Riuniti, Ancona; Elisa Bartolini, MD, Paediatric and Liver Unit, Meyer Children's University Hospital of Florence; Laura Grisotto, MD, Department of Statistics, Informatics and Applications "G. Parenti," University of Florence; Marcello Farallo, MD, Fondazione IRCCS Ca' Granda Ospedale Maggiore Policlinico, Milano; Icilio Dodi, MD, P. Barilla Children's University Hospital of Parma, Parma; Anna Maccabruni, MD, Università degli Studi di Pavia, Pavia; Marco Zaramella, MD, Università degli Studi di Pavia, Pavia; Grazia Bossi, MD, Fondazione IRCCS Policlinico San Matteo, Pavia; Pier Luigi Calvo, MD, Azienda OspedalieraUniversitaria Città della Salute e della Scienza, Torino; Antonio Mazza, MD, Santa Chiara Hospital, Trento; Loredana Lepore, MD, University of Trieste, Trieste; Francesca Barbieri, MD, University of Trieste, Trieste.

\section{REFERENCES}

1. Paul F, Pellegrini S, Uzé G. IFNA2: the prototypic human alpha interferon. Gene. 2015;567:132-137.

2. Lee CK, Jonas MM. Hepatitis C: issues in children. Gastroenterol Clin North Am. 2015;44:901-909.

3. Druyts E, Thorlund K, Wu P, et al. Efficacy and safety of pegylated interferon alfa-2a or alfa-2b plus ribavirin for the treatment of chronic hepatitis $\mathrm{C}$ in children and adolescents: a systematic review and meta-analysis. Clin Infect Dis. 2013;56:961-967.

4. Narciso-Schiavon JL, Schiavon Lde L. Autoantibodies in chronic hepatitis C: a clinical perspective. World J Hepatol. 2015;7:1074-1085.
5. Molleston JP, Mellman W, Narkewicz MR, et al; PEDS-C Clinical Research Network. Autoantibodies and autoimmune disease during treatment of children with chronic hepatitis C. J Pediatr Gastroenterol Nutr. 2013;56:304-310.

6. Pastore F, Martocchia A, Stefanelli M, et al. Hepatitis C virus infection and thyroid autoimmune disorders: a model of interactions between the host and the environment. World J Hepatol. 2016;8:83-91.

7. Hamza I, Eid Y, El-Sayed M, et al. Thyroid dysfunction in chronic hepatitis $\mathrm{C}$ patients treated with the combined pegylated interferon-ribavirin therapy. J Interferon Cytokine Res. 2016;36:527-533.

8. Hwang Y, Kim W, Kwon SY, et al. Incidence of and risk factors for thyroid dysfunction during peginterferon $\alpha$ and ribavirin treatment in patients with chronic hepatitis C. Korean J Intern Med. 2015;30:792-800.

9. Andrade LJ, Atta AM, D'Almeida Junior A, et al. Thyroid dysfunction in hepatitis $\mathrm{C}$ individuals treated with interferon-alpha and ribavirin-a review. Braz J Infect Dis. 2008;12:144-148.

10. Indolfi G, Stagi S, Bartolini E, et al. Thyroid function and anti-thyroid autoantibodies in untreated children with vertically acquired chronic hepatitis C virus infection. Clin Endocrinol (Oxf). 2008;68:117-121.

11. Wirth S, Pieper-Boustani H, Lang $\mathrm{T}$, et al. Peginterferon alfa- $2 \mathrm{~b}$ plus ribavirin treatment in children and adolescents with chronic hepatitis $\mathrm{C}$. Hepatology. 2005;41:1013-1018.

12. Jara P, Hierro L, de la Vega A, et al. Efficacy and safety of peginterferonalpha $2 \mathrm{~b}$ and ribavirin combination therapy in children with chronic hepatitis C infection. Pediatr Infect Dis J. 2008;27:142-148.

13. Wirth S, Ribes-Koninckx C, Calzado MA, et al. High sustained virologic response rates in children with chronic hepatitis $\mathrm{C}$ receiving peginterferon alfa-2b plus ribavirin. J Hepatol. 2010;52:501-507.

14. Sokal EM, Bourgois A, Stéphenne X, et al. Peginterferon alfa-2a plus ribavirin for chronic hepatitis $\mathrm{C}$ virus infection in children and adolescents. $J$ Hepatol. 2010;52:827-831.

15. Baker RD, Dee D, Baker SS. Response to pegylated interferon alpha-2b and ribavirin in children with chronic hepatitis C. J Clin Gastroenterol. 2007;41:111-114.

16. Tajiri $\mathrm{H}$, Inui $\mathrm{A}$, Kiyohara $\mathrm{Y}$, et al. Peginterferon alpha-2b and ribavirin for the treatment of chronic hepatitis $\mathrm{C}$ in Japanese pediatric and young adult patients: a survey of the Japan Society of Pediatric Hepatology. Eur J Gastroenterol Hepatol. 2009;21:1256-1260.

17. Schwarz KB, Gonzalez-Peralta RP, Murray KF, et al; Peds-C Clinical Research Network. The combination of ribavirin and peginterferon is superior to peginterferon and placebo for children and adolescents with chronic hepatitis C. Gastroenterology. 2011;140:450-458.e1.

18. Indolfi G, Nebbia G, Cananzi M, et al; the Italian Study Group for Treatment of Chronic Hepatitis C in Children. Kinetic of virologic response to pegylated interferon and ribavirin in children with chronic hepatitis C predicts the effect of treatment. Pediatr Infect Dis J. 2016;35:1300-1303.

19. Resti M, Bortolotti F, Vajro P, et al; Committee of Hepatology of the Italian Society of Pediatric Gastroenterology and Hepatology. Guidelines for the screening and follow-up of infants born to anti-HCV positive mothers. Dig Liver Dis. 2003;35:453-457.

20. Wirth S, Kelly D, Sokal E, et al. Guidance for clinical trials for children and adolescents with chronic hepatitis C. J Pediatr Gastroenterol Nutr. 2011;52:233-237

21. Stagi S, Pucci N, Di Grande L, et al. Increased incidence of thyroid dysfunction and autoimmunity in patients with vernal keratoconjunctivitis. Int J Endocrinol. 2014;2014:804870.

22. Stagi S, Bindi G, Neri AS, et al. Thyroid function and morphology in patients affected by Williams syndrome. Clin Endocrinol (Oxf). 2005;63:456-460.

23. Stagi S, Giani T, Simonini G, et al. Thyroid function, autoimmune thyroiditis and coeliac disease in juvenile connective tissue diseases. Clin Exp Rheumatol. 2005;23:277.

24. Surks MI, Ortiz E, Daniels GH, et al. Subclinical thyroid disease: scientific review and guidelines for diagnosis and management. JAMA 2004;291:228-238.

25. Stagi S, Giani T, Simonini G, et al. Thyroid function, autoimmune thyroiditis and coeliac disease in juvenile idiopathic arthritis. Rheumatology (Oxford). 2005;44:517-520.

26. Feld JJ, Foster GR. Second generation direct-acting antivirals - Do we expect major improvements? J Hepatol. 2016;65(1 suppl):S130-S142.

27. Balistreri WF, Murray KF, Rosenthal P, et al. The safety and effectiveness of ledipasvir-sofosbuvir in adolescents 12 to 17 years old with hepatitis $\mathrm{C}$ virus genotype 1 infection. Hepatology. 2017;66:371-378. 
28. FDA News Release. FDA Approves Two Hepatitis C Drugs for Pediatric Patients [FDA web site]. 2017. Available at: https://www.fda.gov/newsevents/newsroom/pressannouncements/ucm551407.htm. Accessed June 21, 2017.

29. Serranti D, Indolfi G, Resti M. New treatments for chronic hepatitis C: an overview for paediatricians. World J Gastroenterol. 2014;20:15965-15974.

30. Terrault NA, Bzowej NH, Chang KM, et al; American Association for the Study of Liver Diseases. AASLD guidelines for treatment of chronic hepatitis B. Hepatology. 2016;63:261-283.
31. European Association for the Study of the Liver. EASL clinical practice guidelines: management of chronic hepatitis B virus infection. J Hepatol. 2012;57:167-185.

32. Sokal EM, Paganelli M, Wirth S, et al; European Society of Pediatric Gastroenterology, Hepatology and Nutrition. Management of chronic hepatitis $\mathrm{B}$ in childhood: ESPGHAN clinical practice guidelines: consensus of an expert panel on behalf of the European Society of Pediatric Gastroenterology, Hepatology and Nutrition. J Hepatol. 2013;59:814-829. 